\title{
Correlation of PU.1 and signal regulatory protein $\alpha 1$ expression in PU.1 transgenic K562 cells
}

\author{
YUKO ISEKI $^{1}$, MASAKO NAKAHARA ${ }^{2}$, MAKOTO KUBO $^{3}$, FUMIYA OBATA $^{3}$, \\ HIDEO HARIGAE $^{4}$ and SHINICHIRO TAKAHASHI ${ }^{1,2}$
}

\begin{abstract}
${ }^{1}$ Division of Molecular Hematology, Kitasato University Graduate School of Medical Sciences, Sagamihara; Divisions of ${ }^{2}$ Hematology and ${ }^{3}$ Immunology, Kitasato University School of Allied Health Sciences, Sagamihara; ${ }^{4}$ Department of Hematology and Rheumatology, Tohoku University Graduate School of Medicine, Sendai, Japan
\end{abstract}

Received September 7, 2011; Accepted October 20, 2011

DOI: $10.3892 /$ ijmm.2011.827

\begin{abstract}
PU.1 is a key transcription factor for hematopoiesis and the reduction of this protein expression plays important roles in various hematological malignancies. To identify PU.1 downstream target genes, we recently reported dual microarray analyses, using PU.1 knockdown K562 (K562PU.1KD) cells stably expressing short inhibitory RNAs versus control cells and PU.1-overexpressing K562 (K562PU.1OE) cells versus control cells. Several PU.1 candidate target genes, including cell surface receptor, signal regulatory protein (SIRP) $\alpha 1$, were identified. In this study, we revealed that the expression of SIRP $\alpha 1$ is positively correlated with the expression of PU.1 in various K562PU.1KD and K562PU.1OE cells, shown by realtime PCR and flow cytometry analyses. SIRP $\alpha 1$ is a negative regulator of signaling and its reduced expression is considered to play a role in the pathogenesis of hematological malignancies through the activation of downstream signaling pathways. By comparing 3 different clones of K562PU.1KD cells to their controls, we found constitutive phosphorylation of the extracellular signal-regulated kinase (ERK), but not of Akt, in these cells. Taken together, the down-regulation of PU.1 expression suppresses the expression of SIRP $\alpha 1$, which may play a role in the aberrant activation of ERK in these cells.
\end{abstract}

\section{Introduction}

PU.1 is a member of the Ezb transformation-specific sequence (Ets) family of transcription factors and is expressed in granulocytic, monocytic and B-lymphoid cells $(1,2)$. PU.1 deficient

Correspondence to: Dr Shinichiro Takahashi, Division of Molecular Hematology, Kitasato University Graduate School of Medical Sciences, 1-15-1 Kitasato, Minami-ku, Sagamihara, Kanagawa, 252-0373, Japan

E-mail: shin@kitasato-u.ac.jp

Key words: PU.1, signal regulatory protein $\alpha 1$, extracellular signalregulated kinase, hematological malignancies mice exhibit defects in the development of neutrophils, macrophages and B cells $(3,4)$. It has been reported that mice carrying hypomorphic PU.1 alleles that reduce PU.1 expression, to 20\% of normal levels, developed acute myeloid leukemia (AML) (5). Moreover, down-regulation of PU.1 has been reported to play a role in the pathogenesis of multiple myeloma (6) and is related to a poor prognosis in myelodysplastic syndrome (7). Therefore, characterizing the function and identifying the target genes of PU.1 are important for understanding the molecular biology of hematopoiesis and oncogenesis.

To identify target gene(s) regulated by PU.1, we previously employed dual microarray analyses to compare PU.1-knockdown K562 cells (K562PU.1KD) stably expressing PU.1 siRNAs versus control cells and PU.1 overexpressing K562 cells (K562PU.1OE) versus control cells. From these analyses, we identified metallothionein and vimentin as direct target genes negatively regulated by PU.1 (8). In addition to these, several genes, including signal regulatory protein (SIRP) $\alpha 1$, were identified as candidate target genes, suppressed by PU.1 down-regulation and induced by PU.1 up-regulation (8).

SIRP $\alpha 1$ is a cell surface receptor expressed predominantly in monocytes, granulocytes, dendritic cells, and their precursors, as well as in bone marrow hematopoietic stem cells $(9,10)$. SIRP $\alpha 1$ is also termed Src homology 2 domaincontaining phosphatase (SHP) substrate-1 (SHPS-1), brain immunoglobulin-like molecule with tyrosine-based activation motif (BIT), p84 and macrophage fusion receptor. In humans, there are at least 15 members designated as SIRPs (9). They consist of 2 subtypes distinguished by the presence or absence of a cytoplasmic domain containing immunoreceptor tyrosinebased inhibition motifs (9). SIRP $\alpha 1$ is the most characterized member of the human SIRP family. Its overexpression leads to a reduced responsiveness to tyrosine kinase ligands, such as epidermal growth factor, platelet-derived growth factor and insulin (11). Therefore, SIRP $\alpha 1$ is considered as a negative regulator of signaling.

In this study, we examined whether the expression of PU.1 is indeed correlated with the expression of SIRP $\alpha 1$ in PU.1 transgenic K562 cells. As a result, we clearly revealed that the expression of SIRP $\alpha 1$ is tightly related to the level of PU.1 expression in these cells. Furthermore, we revealed aberrant signaling in K562PU.1KD cells. 


\section{Materials and methods}

Cell culture and generation of PU.1 knockdown cells. K562PU.1KD cells (2-10 and 3-10) and their control cells (vec 5 and vec 6 ) were used as previously described $(8,12)$. K562PU.1OE cells (A2 and H8) and their controls (V1 and V2) (8) were also employed for this study. To generate more K562PU.1KD cells (3-12), PU.1 siRNA expression vectors were transfected into K562 cells by electroporation as described previously $(8,12)$. In addition, we employed pcPURU6ßiSTOP vector (Takara) as a control to generate another control line, vec 4 . K562 clones stably transfected with the PU.1 siRNA vectors and the PU.1 expression vector were maintained with $1 \mu \mathrm{g} / \mathrm{ml}$ puromycin (for siRNA expression vectors) or $400 \mu \mathrm{g} / \mathrm{ml}$ neomycin (for the PU.1 expression vector) containing RPMI with $10 \%$ fetal bovine serum.

mRNA expression analysis. cDNAs were prepared from cells using reverse transcriptase (Superscript II; Invitrogen, Carlsbad, CA). Quantitative PCR was performed using Quantitect SYBRGreen PCR reagent (Qiagen, Miami, FL) following the manufacturer's protocol employing an Opticon mini real-time PCR instrument (Bio-Rad) as previously described (13). Sequences of the primers were: SIRP $\alpha 1$ forward, 5'-CAG TAACATCACCCCAGCA-3' and reverse, 5'-GTCCACGTT GGTCTGGAAGT-3'; PU.1 forward, 5'-GTGCCCTATGACA ACGGATCT-3' and reverse, 5'-GAAGCTCTCGAACTCG CTGT-3' (13); glyceraldehyde-3-phosphate dehydrogenase (GAPDH) forward, 5'-GAAGGTGAAGGTCGGAGT-3' and reverse, 5'-GAAGATGGTGATGGGATTTC-3' (13). Thermal cycling conditions for PU.1 were: step $1,95^{\circ} \mathrm{C}$ for $15 \mathrm{~min}$; step $2,95^{\circ} \mathrm{C}$ for $15 \mathrm{sec}$; step $3,60^{\circ} \mathrm{C}$ for $1 \mathrm{~min}$. Steps $2-3$ were repeated for 35 cycles. The conditions for SIRP $\alpha 1$ were, step 1 , $95^{\circ} \mathrm{C}$ for $15 \mathrm{~min}$; step $2,95^{\circ} \mathrm{C}$ for $30 \mathrm{sec}$; step $3,50^{\circ} \mathrm{C}$ for $30 \mathrm{sec}$; step $4,72^{\circ} \mathrm{C}$ for $30 \mathrm{sec}$. Steps $2-4$ were repeated for 35 cycles. The conditions for GAPDH were, step $1,95^{\circ} \mathrm{C}$ for $15 \mathrm{~min}$; step $2,95^{\circ} \mathrm{C}$ for $30 \mathrm{sec}$; step $3,55^{\circ} \mathrm{C}$ for $30 \mathrm{sec}$; step $4,72^{\circ} \mathrm{C}$ for $30 \mathrm{sec}$. Steps $2-4$ were repeated for 35 cycles. The copy number of each sample was calculated as previously described (14).

Surface marker expression analysis by flow cytometry. For flow cytometry analysis, the cells were incubated with PE-conjugated mouse anti-human CD172 $\alpha$ (SIRP1 $\alpha$ ) (BioLegend, San Diego, $\mathrm{CA}$ ) or PE mouse IgG (BioLegend), lysed with FACSlysis solution (Becton-Dickinson, Mountain View, CA) and applied to a Beckman Coulter Epics XL flow cytometer (Beckman Coulter, Switzerland), as described previously (15).

Western blot analysis. The number of the cells was adjusted in each sample at the day before experiment. Then, the day of the experiment, $1 \times 10^{7}$ cells were collected and lysed in buffer A [10 mM HEPES, $10 \mathrm{mM} \mathrm{KCl}, 1.5 \mathrm{mM} \mathrm{MgCl}_{2}, 1 \mathrm{mM}$ $\mathrm{Na}_{3} \mathrm{VO}_{4}, 0.5 \mathrm{mM}$ DTT, $1 \mathrm{X}$ protease inhibitor cocktail (Roche, Indianapolis, IN)] for $10 \mathrm{~min}$ on ice. After $1,300 \mathrm{x}$ g centrifugation for $10 \mathrm{~min}$, the resulting supernatants were collected as cytosol extracts to examine the signaling molecules. The pellets were washed by buffer B (20 mM HEPES, $420 \mathrm{mM} \mathrm{NaCl}$, $25 \%$ glycerol, $1.5 \mathrm{mM} \mathrm{MgCl}, 0.2 \mathrm{mM}$ EDTA, $0.5 \mathrm{mM}$ DTT, $1 \mathrm{mM} \mathrm{Na}_{3} \mathrm{VO}_{4}, 1 \mathrm{X}$ protease inhibitor cocktail), suspended and incubated on ice for $10 \mathrm{~min}$, centrifuged at $8,000 \mathrm{xg}$
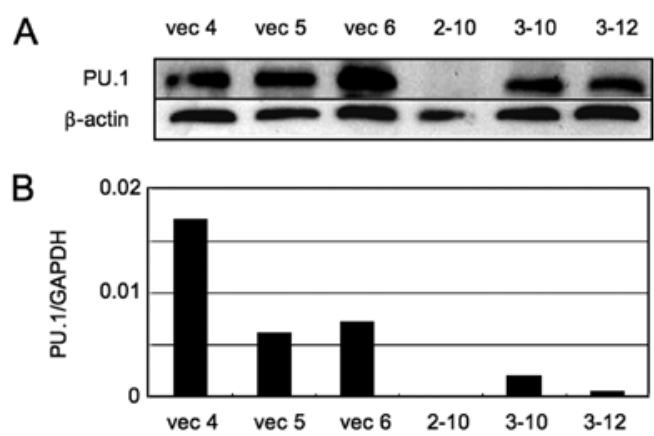

Figure 1. The expression of PU.1 in K562PU.1KD cells. (A) Western blot analysis. Equal amounts of soluble proteins were loaded in each lane and blotted for PU.1 and $\beta$-actin. 2-10, 3-10 and 3-12 cells are K562PU.1KD cells, whereas vec 4, vec 5 and vec 6 cells are their controls. (B) Expression levels of PU.1 in K562PU.1KD cells. Results of real-time quantitative PCR. PU.1 gene transcript level was adjusted by the expression of GAPDH and the relative levels are shown.

A

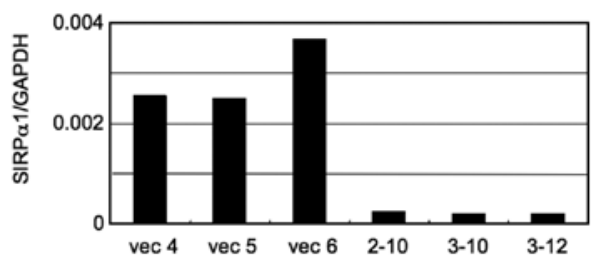

B

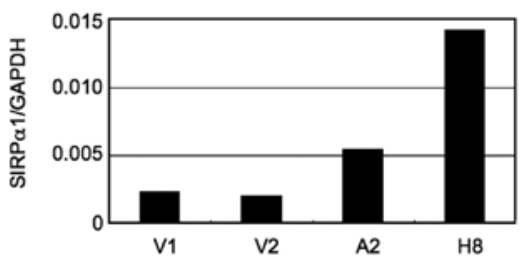

Figure 2. mRNA expression levels of SIRP $\alpha 1$ in PU.1 transgenic K562 cells. Results of real time quantitative PCR in (A) K562PU.1KD cells and their controls, and (B) K562PU.1OE cells (A2,H8) and their controls (V1,V2). SIRP $\alpha 1$ gene transcript level was adjusted by the expression of GAPDH and the relative levels are shown. The data presented were obtained from 3 independent PCR reactions and reproducibility was confirmed by independent real-time PCR from different batches of cDNA.

for $15 \mathrm{~min}$ and supernatants were collected, representing the nuclear extract to examine the expression of PU.1. Proteins (20-30 $\mu \mathrm{g})$ were separated by SDS-PAGE, transferred to Hybond-P PVDF membranes (GE Healthcare, Piscataway, NJ) and immunoblotted as described previously (16). To examine the expression of PU.1, rabbit polyclonal anti-PU.1 (Santa Cruz Biotechnology, Santa Cruz, CA) and mouse monoclonal anti- $\beta$ actin (Sigma, St. Louis, MO) antibodies were used. To examine the signaling, anti-phospho-ERK rabbit polyclonal, anti-phospho-Akt rabbit monoclonal and anti-total-Akt rabbit monoclonal antibodies were purchased from Cell Signaling Technology (Beverly, MA). Anti-total-ERK mouse monoclonal was obtained from BD Biosciences (San Jose, CA).

\section{Results}

Establishment of PU.1-knockdown K562 cells. We previously established K562PU.1KD cells (2-10 and 3-10), stably 
A
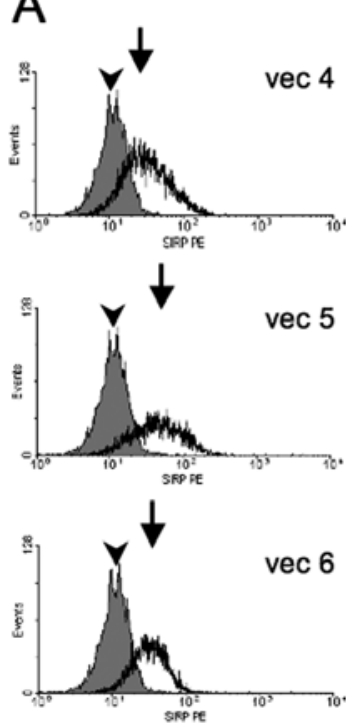
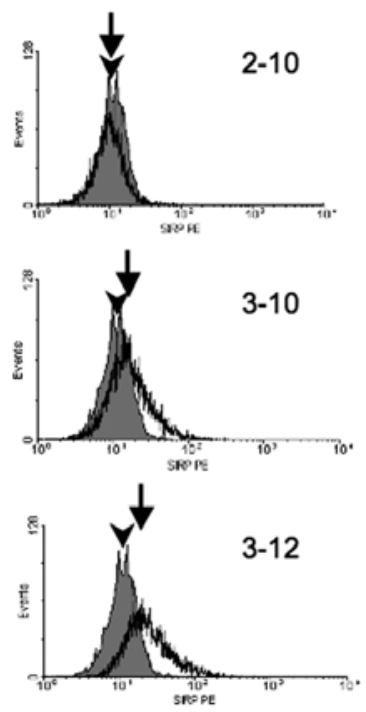

B
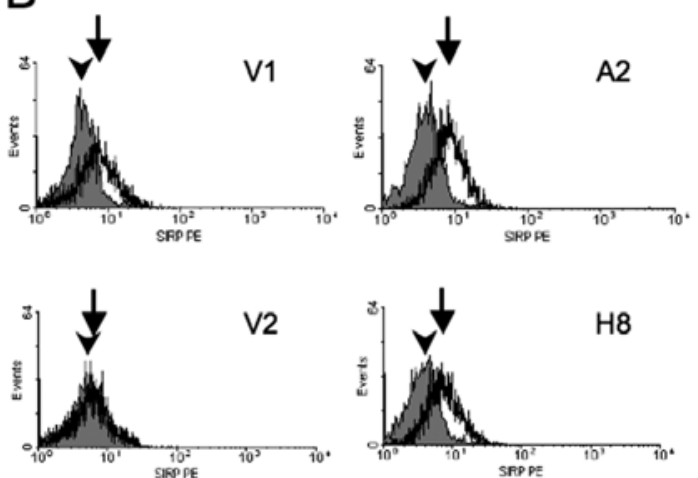

Figure 3. Surface expression levels of SIRP 1 in PU.1 transgenic K562 cells. Flow cytometry analysis of SIRP 1 1 expression on (A) K562PU.1KD, (B) K562PU.1 OE and their control cells. Arrows indicate the SIRP $\alpha 1$ expression while arrowheads indicate the control PE-IgG.

A

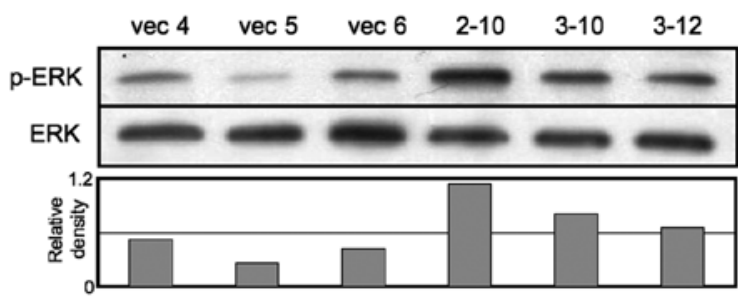

B

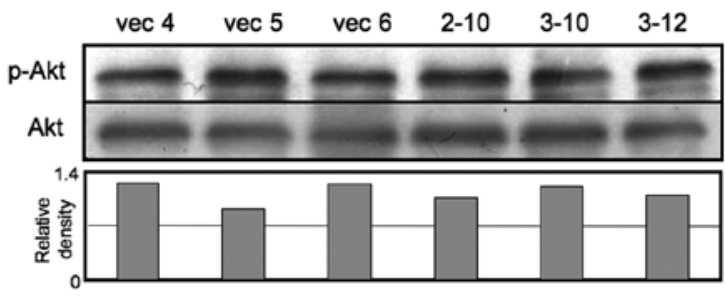

Figure 4. Down-regulation of PU.1 results in the constitutive phosphorylation of ERK. Phosphorylation levels of (A) ERK, and (B) Akt examined by Western blotting. The membranes were first probed with (A) an anti-phospho-ERK and (B) an anti-phospho-Akt rabbit polyclonal antibody. Next, the membranes were stripped and reprobed with (A) anti-total-ERK and (B) anti-total Akt mouse monoclonal antibody to verify equal protein loading. Relative density (lower panels) was obtained from the density of the p-ERK or p-Akt band divided by that of total-ERK or total Akt-band, respectively, measured and quantified by Image J1.36b software (http://rsb.info.nih.gov/ij/).

expressing PU.1 siRNAs, and their control, scrambled siRNA vector transfected vec 5 and vec 6 cells $(8,12)$. To elucidate the relations between PU.1 and SIRP $\alpha 1$, we first attempted to isolate more K562PU.1KD cells and their control cells. Fig. 1 demonstrates the protein (Fig. 1A) and mRNA (Fig. 1B) expressions of PU.1 in K562PU.1KD cells including newly established K562PU.1KD, 3-12 cells. We observed the lowest expression of PU.1 in 2-10 cells and modest suppression in 3-10, and 3-12 cells, compared to their controls, vec 5, vec 6 cells and newly isolated vec 4 cells.
Expression of SIRP 1 in K562PU.1KD and OE cells. The mRNA expression of SIRP $\alpha 1$ in these cells was examined by real-time PCR (Fig. 2). Compared to their control cells (vec 4, vec 5, vec 6), expression of SIRP $\alpha 1$ decreased in all of the PU.1KD cells (2-10, 3-10, 3-12) examined (Fig. 2A). In contrast, SIRP $\alpha 1$ expression significantly increased in K562PU.1OE (A2,H8) cells (Fig. 2B). We then examined the surface expression of SIRP $\alpha 1$ in these PU.1 transgenic K562 cells by flow cytometry analysis. SIRP $\alpha 1$ expression was significantly suppressed in all K562PU.1KD cells (Fig. 3A), whereas it was induced in K562PU.1OE cells (Fig. 3B). These results suggest that the expression of SIRP $\alpha 1$ is positively correlated with the expression of PU.1 in various PU.1 transgenic K562 cells.

Constitutive activation of ERK in K562PU.1 KD cells. The down-regulation of SIRP $\alpha 1$ leads to the activation of downstream signaling pathways $(9,15)$. We previously demonstrated that, by using fewer lines of K562PU.1KD cells, ERK is constitutively activated in these cells (12). To ascertain the activation of downstream signaling pathways by the down-regulation of PU.1, in this study, we employed 3 different K562PU.1KD cells for Western blot analysis. ERK was constitutively phosphorylated in all of the K562PU.1KD cells examined (Fig. 4A). Additionally, there were no differences in Akt phosphorylation in K562PU.1KD compared to control cells (Fig. 4B). Taken together, the down-regulation of PU.1 expression suppresses the expression of SIRP $\alpha 1$, which may play a role in the aberrant activation of ERK in these cells.

\section{Discussion}

It was reported that SIRP $\alpha 1$ expression is significantly reduced in myeloid cells from different leukemias. Seiffert et al (10) reported that all tested myeloid chronic myeloid leukemia blasts (4 of 4) and almost half of the analyzed AML samples (26 of 59) were negative for SIRP $\alpha 1$. In addition, they reported 
that the blasts of 15 of 59 AML samples expressed reduced SIRP $\alpha 1$ levels. We recently identified that the suppression of SIRP $\alpha 1$, by the stable transfection of SIRP $\alpha 1$ siRNA into K562 cells, resulted in the significant phosphorylation of ERK and activation of several downstream signaling pathways (15). Together with these, reduced expression of SIRP $\alpha 1$ may play a role in the mechanisms of leukemogenesis through the aberrant activation of downstream signaling pathways.

SIRPs comprise a family of cell surface receptors that regulate signal transduction through tyrosine kinase receptors by interacting with SHP-1 and SHP-2 (11). SHP-1 tyrosine phosphatase is the key negative regulator for numerous signaling pathways. SHP-1 is expressed primarily in the hematopoietic and epithelial cells. SHP-1 acts in the immune and other hematopoietic cells by inhibiting signaling through receptors for cytokines, growth factors, and chemokines as well as receptors directly involved in the immune responses and programmed cell death $(17,18)$. SHP-1 acts as tumor suppressor and loss of its expression has been identified in the whole spectrum of lymphoid and myeloid cell malignancies (19-21). Recently, it was reported that SHP-1 is directly regulated by PU.1 and suppressed by the PU.1 down-regulation (22). They also revealed that the transient siRNA transfection of PU.1 into Burkitt's lymphoma-derived BJAB cells resulted in the increment of the total intracellular phosphotyrosine (22). In addition, we previously identified that Annexin 1 is induced by the down-regulation of PU.1 (12). Annexin 1 is a calcium- and phospholipids-binding protein and increased expression leads to the constitutive activation of ERK $(12,23)$. Collectively, the reduced expression of PU.1 plays a role in the mechanisms of leukemogenesis thorough the activation of downstream signaling pathways, by modulating the expressions of various genes including SIRP $\alpha 1$, SHP-1 and Annexin 1.

Although genetic alterations affecting the functions of transcription factors that regulate myeloid maturation play important roles in leukemogenesis, inappropriate mitogenactivated protein kinase (MAPK) activation may also play a role in leukemic transformation (24). The Ras-ERK-MAPK pathways and phosphoinositide 3-kinase (PI3K)-Akt pathways are frequently activated in hematological malignancies (25-27). Up-regulation of the MAPK network in AML has been reported to arise through several mechanisms, including the Fms-like tyrosine 3 kinase receptor, c-kit and Ras mutations frequently detected in AML $(14,28-30)$. Our current study sheds light to the fact that the down-regulation of PU.1 transcription factor plays a role in the aberrant induction of ERK-MAPK pathway. Further clarifying the molecular pathways related to PU.1 lead to better understanding of pathogenesis of AML and other hematological malignancies.

\section{Acknowledgements}

The author wish to thank Dr Toshio Okazaki and Dr Takashi Satoh for assistance in the laboratory. This study was supported in part by Grants-in-Aid for Scientific Research (no. 23590687) from the Ministry of Education, Science and Culture, Japan, the Takeda Science Foundation, a foundation from Kitasato University School of Allied Health Sciences (Grant-in-Aid for Research Project, nos. 2008-1001, 2009-1001, 2010-1004, 2011-1001).

\section{References}

1. Klemsz MJ, McKercher SR, Celada A, Van Beveren C and Maki RA: The macrophage and B cell-specific transcription factor PU.1 is related to the ets oncogene. Cell 61: 113-124, 1990.

2. Chen HM, Zhang P, Voso MT, et al: Neutrophils and monocytes express high levels of PU.1 (Spi-1) but not Spi-B. Blood 85: 2918-2928, 1995.

3. Scott EW, Simon MC, Anastasi J and Singh H: Requirement of transcription factor PU.1 in the development of multiple hematopoietic lineages. Science 265: 1573-1577, 1994.

4. McKercher SR, Torbett BE, Anderson KL, et al: Targeted disruption of the PU.1 gene results in multiple hematopoietic abnormalities. EMBO J 15: 5647-5658, 1996.

5. Rosenbauer F, Wagner K, Kutok JL, et al: Acute myeloid leukemia induced by graded reduction of a lineage-specific transcription factor, PU.1. Nat Genet 36: 624-630, 2004.

6. Pettersson M, Sundstrom C, Nilsson K and Larsson LG: The hematopoietic transcription factor PU.1 is downregulated in human multiple myeloma cell lines. Blood 86: 2747-2753, 1995.

7. Huh HJ, Chae SL, Lee M, et al: CD34, RAB20, PU.1 and GFI1 mRNA expression in myelodysplastic syndrome. Int $\mathbf{J}$ Lab Hematol 31: 344-351, 2009.

8. Imoto A, Okada M, Okazaki T, Kitasato H, Harigae $\mathrm{H}$ and Takahashi S: Metallothionein-1 isoforms and vimentin are direct PU.1 downstream target genes in leukemia cells. J Biol Chem 285: 10300-10309, 2010.

9. Barclay AN and Brown MH: The SIRP family of receptors and immune regulation. Nat Rev Immunol 6: 457-464, 2006.

10. Seiffert M,Cant $C, C$ hen Z, et al: Human signal-regulatory protein is expressed on normal, but not on subsets of leukemic myeloid cells and mediates cellular adhesion involving its counterreceptor CD47. Blood 94: 3633-3643, 1999.

11. Kharitonenkov A, Chen Z, Sures I, Wang H, Schilling J and Ullrich A: A family of proteins that inhibit signalling through tyrosine kinase receptors. Nature 386: 181-186, 1997.

12. Iseki $\mathrm{Y}$, Imoto A, Okazaki T, Harigae $\mathrm{H}$ and Takahashi $\mathrm{S}$ : Identification of annexin 1 as a PU.1 target gene in leukemia cells. Leuk Res 33: 1658-1663, 2009.

13. Inomata M, Takahashi S, Harigae H, Kameoka J, Kaku M and Sasaki T: Inverse correlation between Flt3 and PU.1 expression in acute myeloblastic leukemias. Leuk Res 30: 659-664, 2006.

14. Takahashi S, Harigae H, Ishii KK, et al: Over-expression of Flt3 induces NF-kappaB pathway and increases the expression of IL-6. Leuk Res 29: 893-899. 2005.

15. Maekawa T, Imoto A, Satoh T, Okazaki T and Takahashi S: Induction of $\beta$-catenin by the suppression of signal regulatory protein $\alpha 1$ in K562 cells. Int J Mol Med 27: 865-872, 2011.

16. Hirosawa M, Nakahara M, Otosaka R, Imoto A, Okazaki T and Takahashi S: The p38 pathway inhibitor SB202190 activates MEK/MAPK to stimulate the growth of leukemia cells. Leuk Res 33: 693-699, 2009.

17. Shen SH, Bastien L, Posner BI and Chretien P: A protein-tyrosine phosphatase with sequence similarity to the $\mathrm{SH} 2$ domain of the protein-tyrosine kinases. Nature 352: 736-739, 1991.

18. Yi TL, Cleveland JL and Ihle JN: Protein tyrosine phosphatase containing SH2 domains: characterization, preferential expression in hematopoietic cells, and localization to human chromosome 12p12-p13. Mol Cell Biol 12: 836-846, 1992.

19. Zhang Q, Raghunath PN, Vonderheid E, Odum N and Wasik MA: Lack of phosphotyrosine phosphatase SHP-1 expression in malignant T-cell lymphoma cells results from methylation of the SHP-1 promoter. Am J Pathol 157: 1137-1146, 2000.

20. Oka T, Ouchida M, Koyama M, et al: Gene silencing of the tyrosine phosphatase SHP1 gene by aberrant methylation in leukemias/lymphomas. Cancer Res 62: 6390-6394, 2002.

21. Chim CS, Fung TK, Cheung WC, Liang R and Kwong YL: SOCS1 and SHP1 hypermethylation in multiple myeloma: implications for epigenetic activation of the Jak/STAT pathway. Blood 103: 4630-4635, 2004.

22. Wlodarski P, Zhang Q, Liu X, Kasprzycka M, Marzec M and Wasik MA: PU.1 activates transcription of SHP-1 gene in hematopoietic cells. J Biol Chem 282: 6316-6323, 2007.

23. Alldridge LC, Harris HJ, Plevin R, Hannon R and Bryant CE: The annexin protein lipocortin 1 regulates the MAPK/ERK pathway. J Biol Chem 274: 37620-37628, 1999.

24. Takahashi S: Current findings for recurring mutations in acute myeloid leukemia. J Hematol Oncol 4: 36, 2011.

25. Platanias LC: Map kinase signaling pathways and hematologic malignancies. Blood 101: 4667-4679, 2003. 
26. Milella M, Kornblau SM, Estrov Z, et al: Therapeutic targeting of the MEK/MAPK signal transduction module in acute myeloid leukemia. J Clin Invest 108: 851-859, 2001.

27. Takahashi S: Inhibition of the MEK/MAPK signal transduction pathway strongly impairs the growth of Flt3-ITD cells. Am J Hematol 81: 154-155, 2006.

28. Martelli AM, Nyakern M, Tabellini G, et al: Phosphoinositide 3-kinase/Akt signaling pathway and its therapeutical implications for human acute myeloid leukemia. Leukemia 20: 911-928, 2006 .
29. Takahashi S, McConnell MJ, Harigae H, et al: The Flt3 internal tandem duplication mutant inhibits the function of transcriptional repressors by blocking interactions with SMRT. Blood 103: 4650-4658, 2004.

30. Takahashi S: Downstream molecular pathways of FLT3 in the pathogenesis of acute myeloid leukemia: biology and therapeutic implications. J Hematol Oncol 4: 13, 2011. 